\title{
Strengthening Cultural Awareness of Nursing Students
}

\author{
Barbara Donik \\ University of Maribor, Slovenia \\ barbara.donik@um.si \\ Mateja Lorber \\ University of Maribor, Slovenia \\ mateja.lorber@um.si \\ Majda Pajnkihar \\ University of Maribor, Slovenia \\ majda.pajnkihar@um.si
}

Cultural awareness by nurses is key for ensuring quality and person-centred care, therefore, strengthening cultural awareness in nursing students is very important. By measuring cultural awareness of nursing students and involving them in various educational modules in the field of transcultural nursing, we can influence their cultural awareness and sensitivity. The purpose of the research is to measure the level of cultural awareness in nursing students. A quantitative research approach was used. A descriptive cross-sectional survey was performed. For measuring cultural awareness in nursing students, a Cultural awareness scale (CAS) was used. Descriptive statistical methods and inferential statistics were used. The results showed a moderate level of cultural awareness in nursing students. The results indicated that female nursing students' average cultural awareness was higher than in male nursing students. The gender difference was especially high in the dimension Behaviours/Comfort with interactions. The inter-correlation between dimensions that measured cultural awareness was mostly positive. To ensure culturally competent nursing care, it is necessary to start teaching and gaining cultural competences early in their studies as education in the field of cultural competences represents a strategy for improving the knowledge, attitudes and skills of nursing students.

\section{Introduction}

Nurses are faced with an increased need to care for culturally diverse patients (Seal \& Wiske, 2018). This brings the opportunity for strengthening cultural awareness and sensitivity in nurses. Cultural awareness and cultural sensitivity are the key aspects of cultural competence among nurses (Clinton, 1996; Rew, Becker, Cookston, Khosropour, \& Martinez, 2003; Lonneman, 2015). This 
is crucial component in ensuring quality healthcare and has a large impact on the elimination of disparities arising from the perspectives of cultural diversity (Seal \& Wiske, 2018). In order to develop cultural competencies it is necessary to incorporate the contents of cultural diversity into nursing education (Prosen, 2015; Prosen, Karnjuš, \& Ličen, 2017).

Nurses are aware that they have to consider the patients' differences in age, gender, disability, ethnicity, beliefs, and desires for care, norms, and perspectives. Cultural awareness reflects the respect for cultural needs and beliefs (Conway-Klaassen \& Maness, 2017). This is the ability to understand that the cultural background of an individual affects behaviour and interpersonal relationships (Rew et al., 2003; Giger \& Davidhizar, 2007). The response made by individuals and the ability to critically examine personal bias towards other cultures, lifestyles and beliefs (McElroy, Smith-Miller, Madigan, $\& \mathrm{Li}, 2016)$ is therefore the basis for developing explicit views by nurses and their behaviour towards patients from diverse cultural backgrounds (Steed, 2015; Hall, Lee, Clark, \& Perilla, 2016). This reflects an affective dimension of knowledge and adaptation by nurses of awareness of themselves and others (Chen \& Young, 2012).

\section{Strategies for Strengthening Cultural Awareness of Nursing Students}

A critical component of nursing education, practice and research is therefore an integration of multidimensional domains of cultural awareness in nursing curricula (Rew et al., 2003). The challenge, in preparing nursing students to acquire cultural knowledge, is to evaluate whether nursing students are culturally aware, sensitive and competent.

To increase cultural awareness and improve the cultural competence of nursing students, the educational programs must include different learning activities based on patients' social and physical environments as the socioeconomic and political realities that are affecting the patients' varies, as does their access to healthcare (US Department of Health and Human Services, n. d.; Institute of Medicine, 2002; Agency for Healthcare Research and Quality, 2012; Lonneman, 2015). Students brings their own values, beliefs and behavior pattern in the educational setting. These values influence students' thinking, decisions and actions (Jeffreys, 2016). To improve the self-awareness of nursing students of their own culture, including biases and prejudices, are also critical aspects in the educational process (Institute of Medicine, 2002; Agency for Healthcare Research and Quality, 2012). From this perspective, meeting the needs of students who are culturally diverse is a growing challenge in nursing education (Jeffreys, 2016). 
Lonneman (2015) describe six teaching strategies for increasing cultural awareness of nursing students: (1) strategies (games) for raising issues for racism, classism and privilege, (2) reading assigning journal about personal thoughts, feelings and actions, (3) preparing personal history reflection paper and prepare the analysis of personal critical incidents, (4) using different video materials about how racism, classism, and other social factors effects on people's health, (5) performing an interview with someone from vulnerable population and preparing a written report, (6) discussions and purposeful exploration of cultural and health disparities and environmental impacts on health.

It is important to encourage critical thinking among nursing students about, own values and beliefs and the influence of lack of understanding of cultural differences on the patient's outcomes. Studies has shown that educational interventions (workshops, training, educational modules) in the field of learning and acquiring nursing student's cultural competences have a major impact on student's cultural awareness, cultural knowledge, cultural understanding and cultural skills (Brathwaite, 2005; Liu, Stone, \& McMaster, 2018). Because nursing students have less clinical experience with patients from diverse cultural backgrounds, Liu et al. (2018) find out that it is necessary to include into nursing educational programs and curriculums different contents that will have impact on cultural respect. Cultural respect is defined as an essential attitude towards patients coming from a diverse cultural background (Cai, Kunaviktikul, Klunklin, Sripusanapan, \& Avant, 2017). It is also important for students to increase the sense of comfort when they come into interaction with culturally diverse patients, as well as to increase awareness of equality treatment and respect for patient's beliefs, values and behaviors (Cai et al., 2017; Liu et al., 2018).

For increasing cultural awareness of nursing students, different methods for teaching can be included into nursing curricula such as: traditional lecture courses, case studies, role playing and using simulations (Grossman, Mager, Opheim, \& Torbjornsen, 2012; Long, 2012; Lonneman, 2015) and gaming (OngFlaherty, Valencia-Garcia, \& Martinez, 2017). During simulation course that include cultural diversity content students can gain experiences in specific cultural needs of patients based on protective characteristics (age, gender, ethnicity, disability, ...), they can improve communication skills and improve ability in cultural assessment.

The purpose of our research was to measure cultural awareness of nursing students. The aim was to find out the level of cultural awareness of University of Maribor, Faculty of Health Studies (UM FHS) nursing students. 
Three research questions were developed: (1) What is the level of cultural awareness of nursing students on UM FHS? (2) How are dimensions of cultural awareness related with gender, year of study and level of nursing program? (3) How dimensions of cultural awareness are correlating?

\section{Methods}

A descriptive cross-sectional research design was used with a convenience sample of University of Maribor Faculty of Health Sciences nursing students $(N=204)$ : undergraduate 1st year $(n=9)$ 2nd year $(n=102)$, 3rd year $(n=$ $85)$, postgraduates $(n=8)$. Participation rate was $38.9 \%$. Data was collected during summer semester. Before or during the collection of data students haven't received any transcultural educational intervention or course. A combination of online and paper questionnaire was administrated. The data was collected between 17th of May and 31st of August 2018.

\section{Instrument}

For measuring cultural awareness of nursing students, we used Cultural awareness tool (CAS) which was developed by Rew, Becker, Cookston, Khosropour, and Martinez (2003). CAS consisted of 36 items. According to the authors of CAS there are 5 different dimensions that best fit the data. Those are: General Education Experience, Cognitive Awareness, Research Issues, Behaviour/Comfort with Interactions, and Patient Care/Clinical Issues (Rew et al. 2003). The questionnaire was translated into Slovene language by two researchers (BD, $\mathrm{ML})$. For ensuring semantic equivalence we performed the back translation by a professional translator. The evidence of the reliability of the questionnaire (internal consistency) was computed with Cronbach's alpha coefficient, which was 0.51 .

\section{Ethical Considerations}

Permission from University of Maribor, Faculty of Health Studies Ethic Committee was obtained before the study. Students were informed of the purpose of the study. On each questionnaire (paper and online version) there was short description of purpose of the study and other information related to the confidentiality. Those who have been asked to fulfil the paper form of the questionnaire returned them into the sealed envelope. The link with online questionnaire was distributed via social media (Facebook group profiles of each study year).

The anonymity of the participants to the researchers was obtained, because there were no identifying data collected. 
Table 1 Sample Demographics

\begin{tabular}{llrr}
\hline Demographic characteristics & & $N$ & $\%$ \\
\hline Gender & Female & 179 & 87.7 \\
& Male & 25 & 12.3 \\
\hline Program & 2st year & 9 & 4.4 \\
& 2nd year & 102 & 50.0 \\
& 3rd year & 85 & 41.6 \\
& 1st year postgraduate & 5 & 2.5 \\
& 2nd year postgraduate & 3 & 1.5 \\
\hline Type of study & Full time & 174 & 85.3 \\
& Part time & 29 & 14.2 \\
& Unmarked & 1 & 0.5 \\
\hline Ethnicity & Slovene & 192 & 94.1 \\
& Croatian & 2 & 1.0 \\
& Serbian & 4 & 2.0 \\
& Bosnian & 4 & 2.0 \\
& Macedonian & 1 & 0.5 \\
& Hungarian & 1 & 0.5 \\
\hline Language that is used at home & Slovene & 200 & 98.0 \\
& Serbian & 1 & 0.5 \\
& Macedonian & 1 & 0.5 \\
& Bosnian & 1 & 1.0 \\
\hline
\end{tabular}

\section{Data Analysis}

Data was analysed with IBM SPSS Statistics (version 23.0). Descriptive statistics were computed, for answering the research question the arithmetic mean, Mann-Witney test and Spearman's correlation test were used.

\section{Results}

In overall sample, $(N=204)$ students were included, $87.7 \%$ women and $12.3 \%$ male students. The mean age was 22 years old $(s=3.34)$ ranging from min 19 to 45 years old. The sample represent students among undergraduate nursing program and postgraduate nursing program (Table 1). The findings are limited because of small sample of postgraduate nursing students. There were also small group of respondents that vary regarding ethnicity and use of language at home. Descriptive statistic was used to analyze the demographic data. Our results showed, that students accessed two statements inside the 'Research Issues' dimension as not applicable. Because of missing data, the 'Research Issues' dimension was excluded from further analyzing. 
Table 2 Differences between Gender and Dimensions of Cultural Awareness

\begin{tabular}{|c|c|c|c|c|c|c|}
\hline \multirow[t]{2}{*}{ CAS Dimensions } & \multicolumn{2}{|c|}{ Man } & \multicolumn{2}{|c|}{ Woman } & \multirow[t]{2}{*}{$Z$} & \multirow[t]{2}{*}{$p$} \\
\hline & $\bar{x}$ & $s$ & $\bar{x}$ & $s$ & & \\
\hline General Education Experience & 3.73 & 0.326 & 3.59 & 0.407 & -1.689 & 0.091 \\
\hline Cognitive Awareness & 3.76 & 0.677 & 3.79 & 0.599 & -0.105 & 0.916 \\
\hline Behaviors/Comfort with Interactions & 3.32 & 0.703 & 3.64 & 0.663 & -2.058 & 0.040 \\
\hline Patient Care/Clinical Issues & 3.57 & 0.628 & 3.46 & 0.505 & -0.851 & 0.395 \\
\hline Total & 3.58 & 0.345 & 3.62 & 0.303 & -0.361 & 0.718 \\
\hline
\end{tabular}

Notes Column headings are as follows: $\bar{x}$ - average, $s$ - standard deviation, $Z-$ Mann-Whitney test value, $p$ - statistical significance.

The results showed that the average score of self-assessment for the cultures awareness for nursing students was $3.61(s=0.40)$ (72\% of total score). General Education Experience $3.60(s=0.40)$ (72\% of total score), Cognitive Awareness 3.78 ( $s=0.61$ ) (76\% of total score); Behavior/Comfort with Interaction $3.61(s=0.67)$ ( $72 \%$ of total score); Patient Care/Clinical Issues $3.47(s=0.52)$ (69\% of total score). For undergraduate program $15 t$ year nursing students average score was $3.64(s=0.19)$, 2nd year student average score was 3.59 ( $s$ $=0.33)$ and 3 rd year nursing students average score was $3.59(s=0.32)$. For postgraduate program average cultural awareness score was $3.73(s=0.29)$ and 2 nd year nursing students average score was $3.63(s=0.20)$.

The Mann-Whitney test showed that the differences between gender in the dimension 'Behavior/Comfort with Interaction' are perceived. Female students access dimension Behavior/Comfort statistically significantly higher $(z=-2.058 ; p=0.040$ ) (Table 2).

Table 3 presents the results of differences between part time and full-time nursing students. Part time nursing students access the dimension 'General Education Experience' statistically significantly higher $(z=-1.988, p=0.047)$.

Table 3 Mann-Whitney Test between Dimensions of Cultural Awareness and Type of the Study

\begin{tabular}{|c|c|c|c|c|c|c|}
\hline \multirow[t]{2}{*}{ CAS Dimensions } & \multicolumn{2}{|c|}{ Full time } & \multicolumn{2}{|c|}{ Part time } & \multirow[t]{2}{*}{$Z$} & \multirow[t]{2}{*}{$p$} \\
\hline & $\bar{x}$ & $s$ & $\bar{x}$ & $s$ & & \\
\hline General Education Experience & 3.58 & 0.402 & 3.75 & 0.339 & -1.988 & 0.047 \\
\hline Cognitive Awareness & 3.79 & 0.604 & 3.70 & 0.609 & -0.757 & 0.449 \\
\hline Behaviors/Comfort with Interactions & 3.60 & 0.641 & 3.63 & 0.661 & -0.563 & 0.574 \\
\hline Patient Care/Clinical Issues & 3.48 & 0.497 & 3.41 & 0.661 & -0.279 & 0.780 \\
\hline Total & 3.61 & 0.299 & 3.62 & 0.349 & -0.248 & 0.804 \\
\hline
\end{tabular}

Notes Column headings are as follows: $\bar{x}$ - average, $s$ - standard deviation, $Z$ - Mann-Whitney test value, $p$ - statistical significance. 
Table 4 Inter-Correlation between Measured Dimension of Cultural Awareness Scale

\begin{tabular}{lrrrr}
\hline CAS Dimensions & $(1)$ & $(2)$ & $(3)$ & $(4)$ \\
\hline General Education Experience & 1 & $0.349^{* *}$ & 0.117 & $0.308^{* *}$ \\
Cognitive Awareness & - & 1 & -0.085 & $0.420^{* *}$ \\
Behaviors/Comfort with Interactions & - & - & 1 & $-0.203^{* *}$ \\
Patient Care/Clinical Issues & - & - & - & 1 \\
\hline
\end{tabular}

Notes Column headings are as follows: (1) General Education Experience, (2) Cognitive Awareness, (3) Behaviors/Comfort with Interactions, (4) Patient Care/Clinical Issues. ${ }^{*}$ correlation is significant at the 0.05 level or less. ${ }^{* *}$ Correlation is significant at the 0.001 level or less.

In the Table 4 the inter-correlation between measured dimensions in CAS are performed. Spearman's correlation test was performed. The results showed that there is statistically significant weak correlation between the dimensions 'General Education Experience' and 'Cognitive Awareness' ( $r=$ $0.349 ; p<0.001)$ and 'Patient Care/Clinical Issues' $(r=0.308 ; p<0.001)$. There is a moderate correlation between the dimension: 'Cognitive Awareness' and 'Patient Care/Clinical Issues' ( $r=0.420 ; p=0.004)$ and the weak correlation between the 'Behaviors/Comfort with Interactions' and 'Patient Care/Clinical Issues' dimension $(r=0.203 ; p<0.001)$.

\section{Discussion}

The purpose of this study was to measure and analyse cultural awareness of nursing students as first step to improve cultural competencies of nursing students in UM FHS. Results showed that nursing students assessed their level of cultural awareness as moderate (the total average value was 3.61 out of max. 5). According to Starr Tate (2016) slightly lower average mean scores show that there is a space and a need for improvement of student's cultural awareness and cultural competency education courses. To develop cultural awareness, nursing students should have the opportunity to increase their knowledge in transcultural aspect of care. The key for increasing cultural awareness is to improve the understanding of health inequities and health disparities (Cantey, Randolph, Molloy, \& Cary, 2017). It is also crucial that nursing students raise their awareness on the communication and cultural beliefs of people (Goodman, Edge, Agazio, \& Prue-Owens, 2015). There are different strategies to promote cultural awareness and competency within nursing programs (Eshleman \& Davidhizar, 2006). Lonneman (2015) and Long (2012) state that current approaches for increasing cultural competencies are lectures, group discussion, written reports, clinical experiences, simulations, role playing, journal keeping, studying abroad and working experiences in 
community settings. It is important to investigate in further research how effective those strategies are. Cultural competence can be increased by including structured cultural content in the nursing curricula (Sargent, Sedlak, \& Martsolf, 2005).

In our study, the results didn't vary between age, level of nursing program, way of study and year of study. Safipour, Hadziabdic, Hultsjö, and BachrachLindström (2017) also find out an insignificant correlation between demographic variables and the level of cultural awareness. In comparison between female nursing students and male nursing students, the results showed statistically significant higher scores accessed by female students in one dimension: 'Behaviors/Comfort with Interactions.' This can be explained by the results of the study (Parlar Kılıç \& Sevinç, 2018) that female students are more sensitive toward cultural differences, have more positive attitudes toward cultural factors, and that they feel more responsible than male students. Also, Yilmaz, Toksoy, Denizci Direk, Bezirgan, and Boylu (2016) find out, that female scored the determinant: respect for cultural differences higher than male.

Testing the inter-correlation between dimensions was important due to investigate how some dimensions vary between. We can conclude that interesting inter-correlations were found. The negative correlation between Cognitive awareness and Behaviors/Comfort was identified. Positive correlation between 'General Education Experience' and 'Patient Care/Clinical Issues' was identified. There is also a moderate correlation between the dimension: 'Cognitive Awareness' and 'Patient Care/Clinical Issues. Hadziabdic, Safipour, Bachrach-Lindström, and Hultsjö (2016) report the negative intercorrelation between those dimensions.

However, there are some limitation of this study that can be outlined. In this research, the number of male participants, 1st year undergraduate nursing students and postgraduate nursing students is smaller, therefore the results must be interpreted wit conscious. Also, due to small sample there was impossible to measure the relation between demographic variables such as ethnicity and language that participants speak at home with cultural awareness score. The nature of research was quantitative, therefore the results can't provide the impact of some variables that can increase cultural awareness of nursing students.

\section{Conclusions}

Culture is having a huge impact on peoples every day live. During undergraduate or postgraduate nursing education, students must achieve an adequate level of competences, also cultural competences. Concept of culture 
diversity care can be learned and adopted in every nursing education curriculum. This will result the high level of student's cultural awareness. In nursing education there is a need that students perform their clinical settings in diverse - intercultural clinical settings. Further researches are needed to measure cultural awareness among nursing students for identifying strategies for improving their cultural awareness and implementing various teaching strategies in nursing educational programs. The main finding of our study, that there is a moderate level of cultural awareness score among nursing students in our faculty, suggests the importance of continuously measuring the level of cultural awareness between nursing students and recognize the important steps in incorporating strategies for improving cultural awareness of students in nursing education.

\section{References}

Agency for Healthcare Research and Quality. (2012). National Healthcare Disparities Report 2011. Rockville, MD: Agency for Healthcare Research and Quality.

Brathwaite, A. E. (2005). Evaluation of a cultural competence course. Journal of Trancultural Nursing, 16(4), 361-369.

Cai, D., Kunaviktikul, W., Klunklin, A., Sripusanapan, A., \& Avant, P. K. (2017). Developing a cultural competence inventory for nurses in China. International Nursing Review, 64(2), 207-214.

Cantey, D. D., Randolph, S. D., Molloy, M. A., \& Cary, M. P. (2017). Studentdeveloped simulations. Journal of Nursing Education, 56(4), 234-246.

Chen, G. M., \& Young, P. (2012). Intercultural communication competence. In A. Goodboy, \& K. Shultz (Eds.), Introduction to communication studies: Translating scholarship into meaningful practice higher education (pp. 175-188). Dobuque, IA: Kendall-Hunt.

Clinton, J. F. (1996). Cultural diversity and health care in America: Knowledge fundamental to cultural competence in baccalaureate nursing students. Journal of Cultural Diversity, 3(1), 4-8.

Conway-Klaassen, J., \& Maness, L. (2017). Critical conversations: Cultural awareness, sensitivity, and competency. Clinical Laboratory Science, 30(1), 34-37.

Eshleman, J., \& Davidhizar, R. E. (2006). Strategies for developing cultural competency in an RN-BSN program. Journal of Transcultural Nursing, 17, 179183.

Giger, J., \& Davidhizar, J. (2007). Trancultural nursing: Assessments and intervention (5th ed.). St. Louis, MO: Mosby.

Goodman, P., Edge, B., Agazio, J., \& Prue-Owens, K. (2015). Cultural awareness nursing care of Iraqi patients. Journal of Trancultural Nursing, 26(2), 395-401.

Grossman, S., Mager, D., Opheim, H. M., \& Torbjornsen, A. (2012). A bi-national 
simulation study to improve cultural awareness in nursing students. Clinical Simulation in Nursing, 8, e341-e346.

Hadziabdic, E., Safipour, J., Bachrach-Lindström, M., \& Hultsjö, S. (2016). Swedish version of measuring cultural awareness in nursing students: Validity and reliability test. BMC Nursing, 15(25). Retrieved from https://bmcnurs .biomedcentral.com/articles/10.1186/s12912-016-0146-6

Hall, E., Lee, S. Y., Clark, P. C., \& Perilla, J. (2016). Social ecology of adherence to hypertension treatment in latino migrant and seasonal farmworkers. Journal of Transcultural Nursing, 27(1), 33-41.

Institute of Medicine. (2002). Unequal treatment: What healthcare providers need to know about racial and ethnic disparities in health care. Washington, DC: National Academies Press.

Jeffreys, M. R. (2016). Teaching cultural competence in nursing and health care. New York, NY: Springer.

Liu, W., Stone, T. E., \& McMaster, R. (2018). Increasing undergraduate nursing students' cultural competence: An evaluation study. Global Health Research and Policy, 3(7), 1-10.

Long, T. B. (2012). Overview of teaching strategies for cultural competence in nursing students. Journal of Cultural Diversity, 19(3), 102-108.

Lonneman, W. (2015). Teaching strategies to increase cultural. Nurse Educator, 40(6), 285-288.

McElroy, J., Smith-Miller, C. A., Madigan, C. K., \& Li, Y. (2016). Cultural awareness among nursing staff at an academic medical center. The Journal of Nursing Administration, 46(3), 146-153.

Ong-Flaherty, C., Valencia-Garcia, D., \& Martinez, D. A. (2017). Effectiveness of gaming in creating cultural awareness. Learning, Culture and Social Interaction, 12, 149-158.

Parlar Kılıç, S., \& Sevinç, S. (2018). The relationship between cultural sensitivity and assertiveness in nursing students from Turkey. Journal of Transcultural Nursing, 29(4), 379-386.

Prosen, M. (2015). Introducing transcultural nursing education: Implementation of transcultural nursing in the postgraduate nursing curriculum. Procedia: Social and Behavioral Sciences, 174, 149-155.

Prosen, M., Karnjuš, I., \& Ličen, S. (2017). Razvijanje medkulturnih kompetenc med študenti zdravstvene nege. In S. Rutar, K. Čotar, T. Štemberger, \& S. Bratož (Ed.), Vidiki internacionalizacije in kakovosti $v$ visokem šolstvu (pp. 139-153). Koper, Slovenia: University of Primorska Press.

Rew, L., Becker, H., Cookston, J., Khosropour, S., \& Martinez, S. (2003). Measuring cultural awareness in nursing students. Journal of Nursing Education, 42(6), 249-257.

Sargent, S. E., Sedlak, C. A., \& Martsolf, D. S. (2005). Cultural competence among nursing students and faculty. Nurse Education Today, 25(3), 214-221. 
Safipour, J., Hadziabdic, E., Hultsjö, S., \& Bachrach-Lindström, M. (2017). Measuring nursing students' cultural awareness: A cross-sectional study among three universities in southern Sweden. Journal of Nursing Education and Practice, 7(1), 107-113.

Seal, N., \& Wiske, M. (2018, 24 May). 5 ways to improve cultural competence in nursing care. Retrieved from https://minoritynurse.com/5-ways-to -improve-cultural-competence-in-nursing-care/

Starr Tate, A. (2016). Culture counts: An analysis of cultural awareness and competency among nursing students (Undergraduate thesis). Gardner-Web University, Boiling Springs, NC.

Steed, M. R. (2015). Cultural competence in certified registered nurse anesthetists (Doctoral dissertation). Walden University, Minneapolis, MN.

US Department of Health and Human Services. (N. d.). Healty people 2020. Retrieved from https://www.healthypeople.gov/sites/default/files/ HP2020Framework.pdf

Yilmaz, M., Toksoy, S., Denizci Direk, Z., Bezirgan, S., \& Boylu, M. (2016). Cultural sensitivity among clinical nurses: A descriptive study. Journal of Nursing Scholarship, 49(2), 1-9.

S. Ličen, I. Karnjuš, \& M. Prosen (Eds.). (2019). Women, migrations and health: Ensuring transcultural healthcare (pp. 187-197).

Koper, Slovenia: University of Primorska Press.

https://doi.org/10.26493/978-961-7055-43-6.187-197 